\title{
Center for Biologics Evaluation and Research
}

National Cancer Institute

\section{Source}

National Cancer Institute. Center for Biologics Evaluation and Research. NCI Thesaurus. Code C82668.

The center within the Food and Drug Administration that regulates biological products for human use under applicable federal laws. It protects and advances the public health by ensuring that biological products are safe and effective and available to those who need them. It also provides the public with information to promote the safe and appropriate use of biological products. 